\title{
Effects of Source Temperature on Thermodynamic Performance of Transcritical Organic Rankine Cycle
}

\author{
Hyung Jong Ko, Se Woong Kim, Chul Ho Han, and Kyoung Hoon Kim
}

\begin{abstract}
Organic Rankine Cycle (ORC) has attracted much attention as a promising technology for an efficient conversion of low-grade energy into electricity. In this study the thermodynamic performance of transcritical ORC with varying source temperature is investigated. The system uses R134a as working fluid and its performance such as the ratio of mass flow rate, specific net work, and thermal and exergy efficiencies are parametrically investigated. Results for the source temperature ranging $160-300^{\circ} \mathrm{C}$ and the reduced turbine inlet pressure (TIP) up to 3 show that the ratio of mass flow rate increases with TIP for fixed source temperature. It is also shown that the specific net work, and thermal and exergy efficiencies increase with TIP in the subcritical region and have a peak in the supercritical region for each source temperature. Thermal efficiency can be raised by adopting supercritical cycle with sufficiently high source temperature.
\end{abstract}

Index Terms-Organic rankine cycle (ORC), source temperature, transcritical, turbine inlet pressure.

\section{INTRODUCTION}

Since the traditional steam Rankine cycle does not give a satisfactory performance in generating electricity from low-grade heat, most of the low-temperature energy sources such as geothermal energy, exhaust gas, biomass combustion, and waste heat are merely discarded. Therefore, it has become an issue how to efficiently convert the low-grade energy into electricity. Chen et al. [1] reviewed on a variety of thermodynamic cycles for the conversion of low-grade heat and the effects of working fluids on the cycle. Organic Rankine cycle (ORC) which uses organic fluids instead of water as working fluid is considered as one of the promising technologies for such purpose because of the great flexibility, high safety and low maintenance requirements [2].

One of the challenges of ORC is the choice of organic working fluid. It should provide a high thermal efficiency and a high utilization of available heat source. Saleh et al. [3] made a thermodynamic screening of pure component working fluids for low-temperature ORC's in view of the thermal efficiency and heat transfer characteristics. Mago et al. [4] presented a performance analysis of different ORC configuration which uses dry organic working fluids. They showed that regenerative ORC gives higher first and second law efficiencies and requires less heat to produce the same

Manuscript received December 5, 2013; revised February 7, 2013.

Hyung Jong Ko, Chul Ho Han, and Kyoung Hoon Kim are with the Department of Mechanical Engineering, Kumoh National Institute of Technology, Gumi, Gyeongbuk 730-701, Korea (e-mail: \{kohj, chhan, khkim\}@kumoh.ac.kr).

Se Woong Kim is with the Department of Mechanical Engineering, Kumoh National Institute of Technology, Gumi, Gyeongbuk 730-701, Korea (e-mail: ksw@kumoh.ac.kr). power.

One of the key features of ORC is the heat transfer characteristics between the source fluid and working fluid. An important limitation of the ORC with pure working fluid is the constant temperature boiling process, which creates a bad thermal match between the fluids. This problem can be circumvented if supercritical cycle or mixture working fluid is used [5]-[9]. When zeotropic mixture working fluid is used, both the boiling and condensing processes take place with varying temperature, thus a better thermal match is created. Chen et al. [6] found that a supercritical Rankine cycle using zeotropic mixture working fluid results a remarkable improvement of thermal efficiency than a conventional ORC. Chen et al. [7] carried out a comparative study of the carbon dioxide transcritical power cycle with an ORC with R123 as working fluid and showed that the transcritical cycle gives a slightly higher power. Baik et al. [8] carried out a power-based comparison between $\mathrm{CO}_{2}$ and R125 trancritical cycles. The latter cycle was recommended for heat sources about $100^{\circ} \mathrm{C}$. Kim and Han [9] investigated the thermodynamic performance of transcritical ORC with and without internal heat exchanger for various working fluids. It was shown that supercritical cycles could provide better performance than subcritical cycles.

In this study, the thermodynamic performance of transcritical ORC with the reduced turbine inlet pressure (TIP) up to 3 is investigated based on the Patel-Teja equation of state and the laws of thermodynamics. The system uses R134a as working fluid and is driven by the sensible heat of source fluid with temperature ranging $160-300^{\circ} \mathrm{C}$. As an exploration to an efficient conversion of source heat into useful work, the performance of cycle such as the ratio of mass flow rate, specific net work, and thermal and exergy efficiencies are parametrically investigated in terms of the source temperature and TIP.

\section{SYSTEM ANALYSIS}

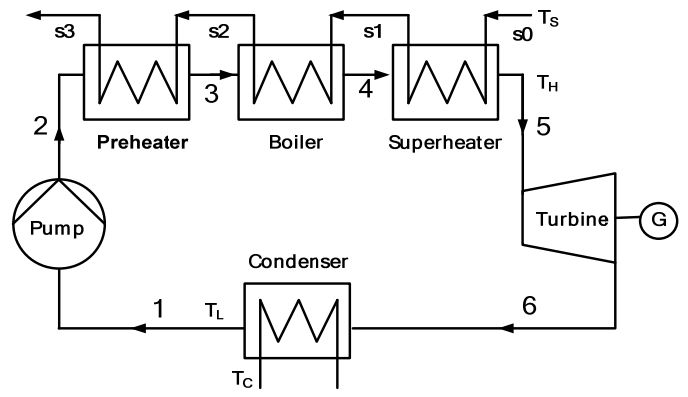

Fig. 1. Schematic diagram of the system.

Fig. 1 shows the schematic diagram of the system 
considered in this study, which is consisted of condenser, pump, heat exchanger (preheater, boiler, superheater), and turbine. Heat is supplied to the system by the source fluid at the heat exchanger and rejected to the coolant at the condenser. The source fluid and working fluid considered in this study are air and R134a, respectively. Water is used as a coolant fluid.

The thermodynamic properties of the working fluid are calculated by the Patel-Teja equation of state [10], [11], which is written as

$$
P=\frac{R T}{v-b}-\frac{a}{v(v+b)+c(v-b)}
$$

Here, $P, T, v$ and $R$ are pressure, absolute temperature, specific volume and gas constant, respectively, and $a, b$, and $c$ are determined from $T$ and the parameters of the critical point. The basic data of the working fluid which are needed to calculate $a, b$, and $c$ are given in TABLE I, where $M, T_{c r}$, $P_{c r}$ and $\omega$ are molecular weight, critical temperature, critical pressure, and acentric factor, respectively [10]-[12]. By the way organic fluids can be classified into three groups by the sign of $\mathrm{dT} / \mathrm{ds}$ on the saturated vapor line, that is, wet, dry, and isentropic fluids. R134a belongs to isentropic fluids [13].

TABLE I: BASIC DATA OF THE WORKING FLUID

\begin{tabular}{ccccc}
\hline \hline Substance & $M(\mathrm{~kg} / \mathrm{kmol})$ & $T_{c r}(\mathrm{~K})$ & $P_{c r}(\mathrm{bar})$ & $\omega$ \\
\hline R134a & 102.031 & 380.00 & 36.90 & 0.239 \\
\hline \hline
\end{tabular}

In order to investigate the thermodynamic performance of the system the following simplifications are utilized. 1) The source fluid is standard air and flows into the system at a constant temperature of $T_{S}$. 2) The working fluid leaves the condenser as saturated liquid at temperature of $T_{L}$. 3) The turbine inlet temperature is lower than $T_{S}$ by $\Delta T_{H}$, that is, $T_{H}=$ $T_{S}-\Delta T_{H}$. 4) The temperature difference between the hot and cold streams in the heat exchanger is higher than a prescribed value of pinch point temperature difference. 5) The heat transfer process in the heat exchanger is modeled using a heat transfer effectiveness. 6) The coolant fluid is water with inlet temperature of $T_{c}$. 7) The pump and turbine have constant isentropic efficiencies of $\eta_{p}$ and $\eta_{t}$, respectively. 8) There is no pressure drop or heat loss in the system. 9) Heat supply and rejection process take place at constant pressure of $P_{H}$ and $P_{L}$, respectively.

When the system is operated as a supercritical cycle, there is no apparent phase change process in the heat changer. Therefore the identification of locations 3 and 4 in Fig. 1 is not possible in this case. However if the system is operated as a subcritical cycle, points 3 and 4 correspond to saturated liquid and saturated vapor, respectively. The thermodynamic states at 1, 2, 5 and 6 are determined as follows. At point 1, the fluid is saturated liquid at temperature $T_{L}$ and the corresponding saturation pressure $P_{L}$ is the low pressure of the system. When the TIP is $P_{H}$, point 2 is determined such that the pressure is $P_{H}$ and the isentropic efficiency of the pump is equal to $\eta_{p}$. At point 5 , the fluid has pressure $P_{H}$ and temperature $T_{H}$. Finally, point 6 is determined such that the turbine has outlet pressure $P_{L}$ and isentropic efficiency $\eta_{t}$.
It is desirable to produce electricity as much as possible with the energy source available in the form of sensible heat. Therefore it is necessary to circulate more working fluid and less coolant fluid for a unit mass flow rate of source fluid, unless the pinch point condition is violated. The ratios of mass flow rate of working fluid and coolant fluid to that of the source fluid, $r_{w f}$ and $r_{c}$, can be determined from the energy balance and the heat transfer effectiveness as follows:

$$
\begin{gathered}
r_{w f}=\frac{\dot{m}_{w f}}{\dot{m}_{s}}=\frac{c_{p s}\left(T_{s}-T_{s 3}\right)}{h_{5}-h_{2}} \\
T_{s 3}=T_{s}-\eta_{h e}\left(T_{s}-T_{2}\right) \\
\min \left(T_{\text {source }}-T_{w f}\right) \geq \Delta T_{P P} \\
r_{c}=\frac{\dot{m}_{c}}{\dot{m}_{s}}=\frac{h_{6}-h_{1}}{c_{p c}\left(T_{c, o u t}-T_{c}\right)} \cdot r_{w f} \\
\min \left(T_{w f}-T_{\text {coolant }}\right)=\Delta T_{P P}
\end{gathered}
$$

where subscripts $w f$, $c$, and $s$ refer to the working fluid, coolant fluid, and source fluid, respectively, and $\dot{m}$ the mass flow rate, $h$ the specific enthalpy, $c_{p}$ the specific heat at constant pressure. $\eta_{h e}$ and $\Delta T_{P P}$ mean the heat transfer effectiveness of the heat exchanger and the pinch point temperature difference.

The rates of heat input and net work production can be calculated from

$$
\begin{gathered}
\dot{Q}_{i n}=\dot{m}_{w f}\left(h_{5}-h_{2}\right) \\
\dot{W}_{n e t}=\dot{W}_{t}-\dot{W}_{p}=\dot{m}_{w f}\left[\left(h_{5}-h_{6}\right)-\left(h_{2}-h_{1}\right)\right]
\end{gathered}
$$

where subscripts $t$ and $p$ refer to the turbine and pump, respectively.

The exergy which is a property of substance is defined as the maximum useful work available when the system evolves reversibly to reach equilibrium with the environment which is said to be in dead state. When a system undergoes a steady state operation, the thermodynamic properties of working fluid at any reference state can be assigned zero. It is convenient to take the ambient condition or dead state as the reference state for the enthalpy, entropy, and exergy. The specific exergy $e$ and the rate of exergy input to the system by source fluid can be calculated as [14]

$$
\begin{gathered}
e=h-h_{0}-T_{0}\left(s-s_{0}\right) \\
\dot{E}_{\text {in }}=\dot{m}_{s} c_{p s}\left\{T_{s}-T_{0}-T_{0} \ln \left(T_{s} / T_{0}\right)\right\}
\end{gathered}
$$

where $s$ is the specific entropy and subscript 0 refers to the dead state

The overall performance of the system can be assessed with the thermal efficiency, $\eta_{t h}$, and the exergy efficiency, $\eta_{e x}$, which are defined as the ratio of net work to heat input and exergy input, respectively, as follows. 


$$
\begin{aligned}
& \eta_{t h}=\dot{W}_{n e t} / \dot{Q}_{i n} \\
& \eta_{e x}=\dot{W}_{n e t} / \dot{E}_{\text {in }}
\end{aligned}
$$

It is to be noted that the exergy efficiency is a measure of how close the system is to a reversibly operating system.

\section{RESULTS AND DISCUSSIONS}

In this study the effect of source fluid temperature on the thermodynamic performance of R134a transcritical cycle is parametrically investigated. The reduced turbine inlet pressure, $P_{R}=P_{H} / P_{c r}$, is varied up to 3 (about 110 bar for $P_{H}$ ), while the temperature ranging from $160^{\circ} \mathrm{C}$ to $300^{\circ} \mathrm{C}$ with an interval of $20^{\circ} \mathrm{C}$ is assumed for the source temperature $T_{S}$. Other basic data for analysis are as follows; $T_{L}=30^{\circ} \mathrm{C}, T_{c}=$ $15^{\circ} \mathrm{C}, T_{0}=15^{\circ} \mathrm{C}, \Delta T_{H}=20^{\circ} \mathrm{C}, \Delta T_{P P}=10^{\circ} \mathrm{C}, \eta_{p}=0.8, \eta_{t}=0.8$, $\eta_{h e}=0.7$. Since it is important in organic Rankine cycles to efficiently convert the sensible heat into electricity, the performance of the system including the mass flow rate of the working fluid and the net power production per unit mass flow rate of source fluid is investigated for the varying values of $P_{R}$ and $T_{S}$.

When the TIP is lower than the critical pressure, the cycle is a subcritical cycle. In this case boiling occurs at constant temperature and there exist clear phase change processes in the heat exchanger. Fig. 2 is an assessment of heat transfer in the heat exchanger, where the percentage of heat transfer in the three sections is figured out. Since the temperature at the exit of superheater is equal to $T_{S}-\Delta T_{H}$, the portion of heat transfer in the superheater is larger for higher $T_{S}$. The rest of heat transfer is used to raise the temperature of working fluid to boiling temperature and then to vaporize it. The former portion is dominant for high TIP, while the latter portion is dominant for low TIP. This is because the boiling temperature increases with $P_{H}$ and the latent heat of vaporization decreases with $P_{H}$. In a limit case of $P_{R}=1$ there is no heat transfer in the boiler. In contrast to the nearly linear dependence in the preheater and boiler, the variation of the percentage heat transfer with respect to $P_{H}$ is relatively small in the superheater.

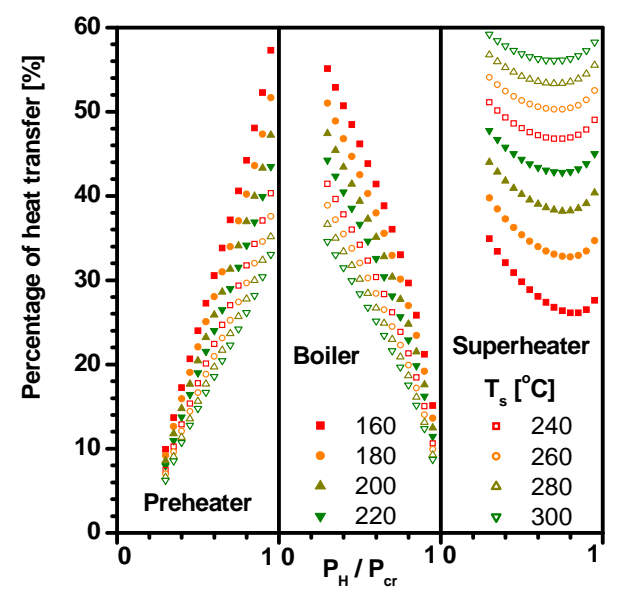

Fig. 2. Percentage of heat transfer in the heat exchanger.

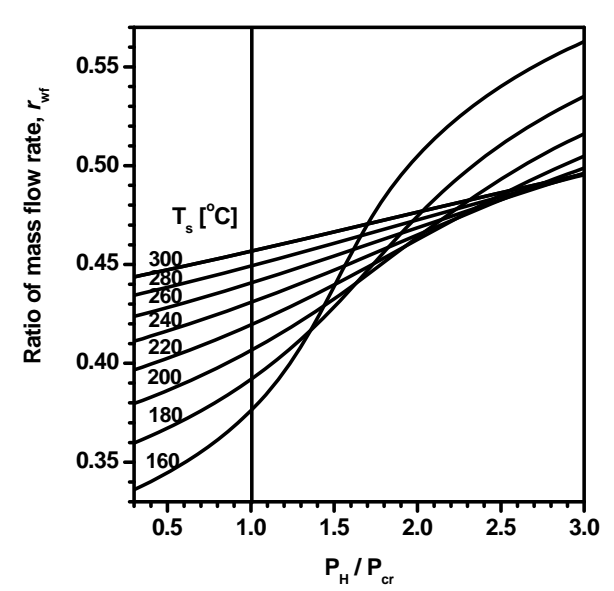

Fig. 3. Ratio of mass flow rate, $r_{w f}$.

Fig. 3 shows the dependence of the ratio of mass flow rate, $r_{w f}$, on the reduced turbine inlet pressure, $P_{R}$, and source temperature, $T_{S}$. Notice a vertical line dividing the subcritical and supercritical cycles. The ratio of mass flow rate of working fluid to that of source fluid increases with $P_{R}$, and the increasing rate becomes higher as the source temperature gets lower. For fixed $P_{R}, r_{w f}$ is larger for higher $T_{S}$ in the subcritical case. However, there exists a reversal of trend in the supercritical region. A higher value of $r_{w f}$ implies that more working fluid could be circulated, which is favorable to produce more power with the same enthalpy drop.

The volume flow rate at the exit of turbine for the production of $1 \mathrm{~kW}$ of net power is shown in Fig. 4 as a function of the reduced TIP for varying source temperature. It is an important factor for the selection of working fluid of the power plant since it is directly related with the size and cost of the turbine. When the system is operated as a subcritical cycle the turbine exit volume flow rate is not sensitive to source temperature and decreases with the TIP. For relatively high source temperatures, this trend extends to the supercritical region in spite of the slowdown of decreasing rate. However the trend changes if the source temperature is relatively low. The volume flow rate begins to increase past a bottom point. It seems, therefore, undesirable to operate a system at high pressure when the source fluid temperature is low.

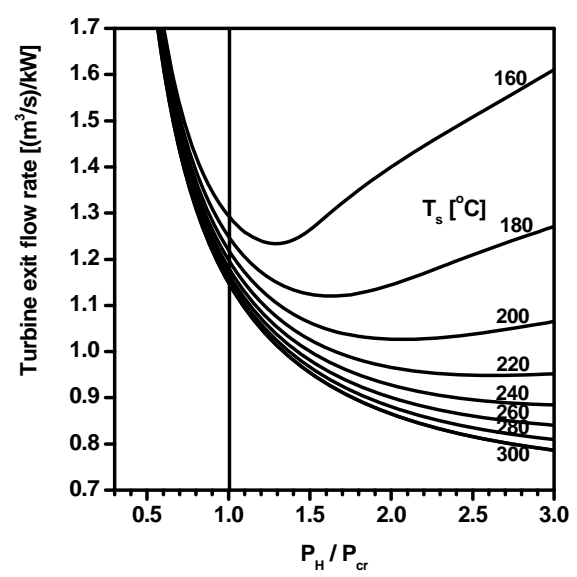

Fig. 4. Turbine exit volume flow rate per unit power production. 
One of the important aspects which must be considered is the specific net power production of a power cycle. In view of the optimal utilization of low-grade heat sources, net work per unit mass of source fluid is an appropriate variable for such purpose and is plotted in Fig. 5 with respect to $P_{R}$ and $T_{S}$. As expected, the net work is larger for higher source temperature for the same TIP irrespective of subcritical or supercritical. When the source temperature is fixed, the net work increases with the TIP in a subcritical region. In a supercritical region the increasing rate slows down and begins to decrease past a peak point. The peak point shifts to the right with the increase of $T_{S}$ although they are invisible for $T_{S}>220^{\circ} \mathrm{C}$ because of the pressure range limitation. It should be noted that the specific net work of the supercritical cycles are several times larger than that of the subcritical cycles.

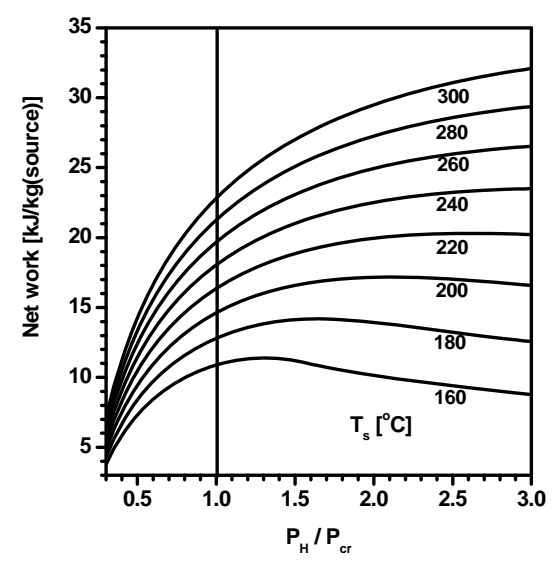

Fig. 5. Net work production per unit mass of source fluid.

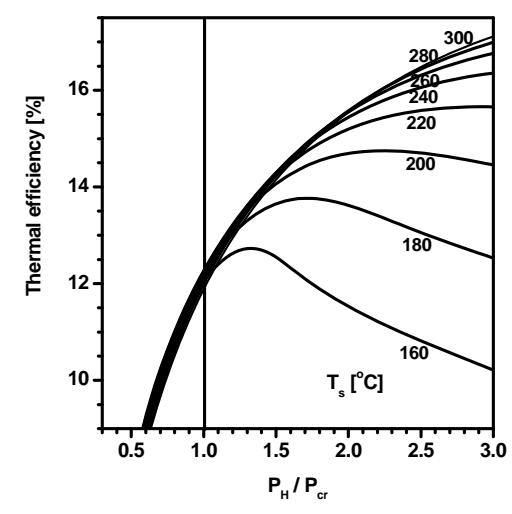

Fig. 6. Thermal efficiency of the cycle.

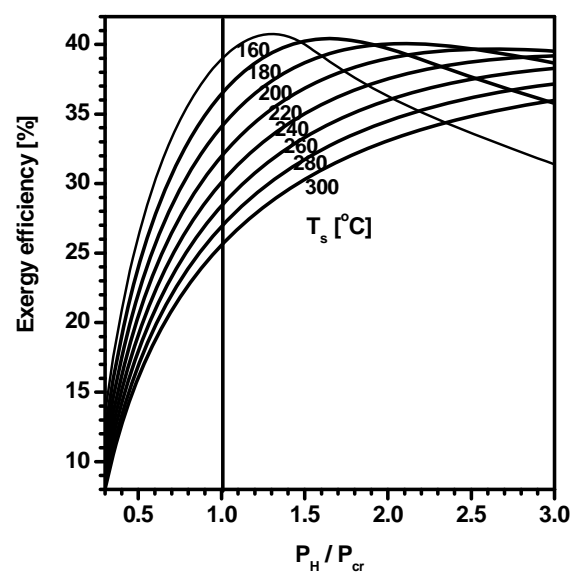

Fig. 7. Exergy efficiency of the cycle.
Finally we will consider the overall performance of the system in terms of the first and second law efficiencies of thermodynamics. Fig. 6 and Fig. 7 show the variations of the thermal efficiency and the exergy efficiency of the cycle, respectively, with respect to $P_{R}$ and $T_{S}$. Thermal efficiency of both subcritical and supercritical cycles, as seen in Fig. 6, is higher if the source temperature is higher. In contrast to a marginal difference in the subcritical cycles, it shows a strong dependence on $T_{S}$ in the supercritical cycles. For each fixed $T_{S}$, thermal efficiency has a peak value in a supercritical region where the peak points shift to the right with increasing $T_{S}$. This behavior is qualitatively similar to that of specific net work. In spite of relatively low value compared to the conventional power cycle, thermal efficiency can be raised by adopting supercritical cycle with sufficiently high source temperature from $12 \%$ maximum to $15-17 \%$.

The exergy efficiency of the cycle is plotted with respect to the reduced turbine inlet pressure and source temperature in Fig. 7. Like specific net work in Fig. 5 and thermal efficiency in Fig. 6, the exergy efficiency increases with the reduced TIP in the subcritical region and attains a peak value and then decreases in the supercritical region for each fixed source temperature. The peak values range from $38 \%$ to $40 \%$ in the pressure range considered. Contrary to thermal efficiency, the exergy efficiency gets lower as source temperature gets higher until the peak points appear. In this range supercritical cycles give higher exergy efficiency than subcritical cycles. Since a high source temperature results in a high exhaust temperature, the recuperation of exhaust heat may contribute to an improvement of exergy efficiency.

\section{CONCLUSIONS}

In this study, the effect of source fluid temperature on the thermodynamic performance of the R134a transcritical cycle is parametrically investigated for the reduced TIP up to 3 . The main results can be summarized as follows.

The ratio of mass flow rate of working fluid to source fluid increases with TIP. For fixed TIP, it is larger for higher source temperature in the subcritical case.

In subcritical cycles the turbine exit volume flow rate is not sensitive to source temperature and decreases with the TIP. It has a bottom point in the supercritical range.

The net work production per unit mass of source fluid is larger for higher source temperature for the same TIP irrespective of subcritical or supercritical. For fixed source temperature, it has a ascending-descending pattern with the TIP.

Thermal efficiency of cycle is higher if the source temperature is higher. For fixed source temperature, it has a peak value in a supercritical region. It can be raised by adopting supercritical cycle with sufficiently high source temperature from $12 \%$ maximum to $15-17 \%$.

The exergy efficiency of cycle increases with the reduced TIP in the subcritical region and has a peak value in the supercritical region for each source temperature.

\section{ACKNOWLEDGMENT}

This research was supported by Basic Science Research 
Program through the National Research Foundation of Korea (NRF) funded by the Ministry of Education, Science and Technology (No. 2012-013929).

\section{REFERENCES}

[1] H. Chen, D. Y. Goswami, and E. K. Stefanakos, “A review of thermodynamic cycles and working fluids for the conversion of low-grade heat," Renewable and Sustainable Energy Reviews, vol. 14, pp. 3059-3067, 2010.

[2] N. A. Lai, M. Wendland, and J. Fisher, "Working fluids for high temperature organic Rankine cycle,” Energy, vol. 36, pp. 199-211, 2011.

[3] B. Saleh, G. Koglbauer, M. Wendland, and H. Fischer, "Working fluids for low-temperature organic Rankine cycle,” Energy, vol. 32, pp. 1210-1221, 2007.

[4] P. J. Mago, L. M. Charma, K. Srinivasan, and C. Somayaji, "An examination of regenerative organic Rankine cycles using dry fluids," Applied Thermal Eng., vol. 28, pp. 998-1007, 2008.

[5] K. H. Kim, C. H. Han, and K. Kim, "Effects of ammonia concentration on the thermodynamic performances of ammonia-water based power cycles,” Thermochimica Acta, vol. 530, pp. 7-16, 2012.

[6] H. Chen, D. Y. Goswami, M. M. Rahman, and E. K. Stefanakos, “A supercritical Rankine cycle using zeotropic mixture working fluids for the conversion of low-grade heat into power," Energy, vol. 36, pp. 549-555, 2011

[7] Y. Chen, P. Lundqvist, A. Johansson, and P. Platell, “A comparative study of the carbon dioxide transcritical power cycle compared with an organic Rankine cycle with R123 as working fluid in waste heat recovery,” Applied Thermal Eng., vol. 26, pp. 2142-2147, 2006.

[8] Y. J. Baik, M. S. Kim, K. C. Chang, and S. J. Kim, "Power-based performance comparison between carbon dioxide and R125 transcritical cycles for a low-grade heat source," Applied Energy, vol. 88, pp. 892-898, 2011.

[9] K. H. Kim and C. H. Han, "Analysis of transcritical organic Rankine cycles for low-grade heat conversion,” Adv. Sci. Lett., vol. 8, pp. 216-221, 2012.

[10] T. Yang, G. J. Chen, and T. M. Guo, "Extension of the Wong- Sandler mixing rule to the three-parameter Patel-Teja equation of state: Application up to the near-critical region,” Chem. Eng. J., vol. 67, pp. 27-36, 1997.

[11] L. D. Gao, Z. Y. Li, S. G. Zhu, and S. G. Ru, "Vapor-liquid equilibria calculation for asymmetric systems using Patel-Teja equation of state with a new mixing rule," Fluid Phase Equilibria, vol. 224, pp. 213219, 2004.

[12] C. L. Yaws, Chemical properties handbook, McGraw- Hill, 1999.

[13] K. H. Kim, "Thermodynamic performance of regenerative organic Rankine cycles,” WASET, vol. 59, pp. 1515-1519, 2011.

[14] K. H. Kim and H. J. Ko, "Exergetical performance assessment of organic Rankine cycle with superheating,” App. Mech. Materials, vol. 234, pp. 69-73, 2012.

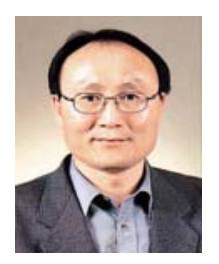

Hyung Jong Ko received the Ph.D. degree in mechanical engineering from Korea Advanced Institute of Science and Technology (KAIST). He is currently a Professor in the Department of Mechanical Engineering at Kumoh National Institute of Technology, Korea. His research interests are in the areas of modeling of simultaneous heat and mass transfer, and analysis of magnetic fluid flow.

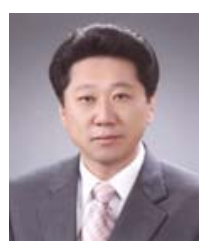

Se Woong Kim received the Ph.D. degree in mechanical engineering from Seoul National University. He is currently a Professor in the Department of Mechanical Engineering at Kumoh National Institute of Technology, Korea. His research interests are in the areas of automotive engineering and new energy system.

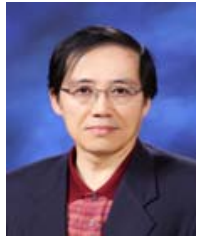

Chul Ho Han received the Ph.D. degree in production engineering from Korea Advanced Institute of Science and Technology (KAIST). He is currently a Professor in the Department of Intelligent Mechanical Engineering at Kumoh National Institute of Technology, Korea. His research interests are in the areas of reliability engineering of systems and evaluation of formability test.

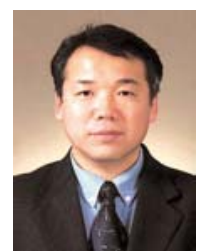

Kyoung Hoon Kim received the Ph.D. degree in mechanical engineering from Korea Advanced Institute of Science and Technology (KAIST). He is currently a Professor in the Department of Mechanical Engineering at Kumoh National Institute of Technology, Korea. His research interests are in the areas of modeling and design of energy systems. 